\title{
Double inclusive cross sections for gluon production in collision of two projectiles on two targets in the BFKL approach
}

\author{
M. A. Braun ${ }^{\text {a }}$ \\ Department of High Energy Physics, Saint-Petersburg State University, 198504 Saint-Petersburg, Russia
}

Received: 9 February 2014 / Accepted: 28 April 2014 / Published online: 9 May 2014

(C) The Author(s) 2014. This article is published with open access at Springerlink.com

\begin{abstract}
Double inclusive cross sections for gluon production in collision of two nucleons with two nucleons are studied in the BFKL approach. Various contributions include emission from the pomerons attached to the participants, from the BFKL interactions between these pomerons and from the intermediate BKP state. The last contribution may be observable, provided the growth with energy of the pomeron contribution is tamed in accordance with unitarity. The possibility of long-range azimuthal correlations due to the BKP state are discussed.
\end{abstract}

\section{Introduction}

In our previous papers [1,2] we have derived the forward scattering amplitude and single inclusive cross section for gluon production for two-projectile-two-target collisions at high energies in the BFKL approach. Their immediate applications are to the cross section for deuteron-deuteron collisions, although this also concerns a part of heavy-nucleusheavy-nucleus collisions due to interaction of two pairs of nucleons. A remarkable result found in [1] is that the cross sections contain a contribution from the intermediate state consisting of four reggeised gluons in the octet colour state between the neighbours (BKP state $[3,4]$ ). In this paper we study double inclusive cross sections for gluon production for the same process. This process presents a special interest in view of experimental observation of the long-range azimuthal correlations in the production of a pair of particles. Note that azimuthal asymmetry in two gluon production has been claimed to be the source of the observed correlation in the framework of the JIMWLK (or colour glass condensate) approach in collision of two heavy nuclei [5]. We shall study the same problem for collision of light nuclei, where the results of [5] are not valid. In contrast to the case of heavy

a e-mail: braun1@ pobox.spbu.ru nuclei, where only approximate treatment of heavy-nucleusheavy-nucleus collisions is possible, the light nucleon case allows a consistent and rigorous study. Thus we are going to see for certain if the initial process of gluon production really leads to the azimuthal asymmetry.

As derived in [1] in the lowest order in $\alpha_{s} N_{c}$, assumed small, the scattering amplitude for the collision of two projectiles on two targets is described by the diagrams shown in Fig. 1.

Diagram 1 corresponds to direct sewing of the two pomerons attached to the projectiles with the two pomerons attached to the targets with redistribution of colour. Diagram 2 describes the situation when the gluons connecting the projectiles and targets once interact between themselves. Diagrams 3 and 4 cover all the rest cases when the exchanged gluons interact at least twice. The state formed between these interactions is the BKP state made of four reggeised gluons. The imaginary part of the forward scattering amplitude is the sum of the contributions from Fig. 1-1-4 and 4:

$D=\sum_{i=1}^{4} D^{(i)}$

The forward amplitude $D^{(1)}$ corresponding to Fig. 1, derived in [1], contains an infrared divergent part. Indeed both of the two pairs of pomerons coupled to the projectiles and targets contain the factor $1 /(E-4 \omega(q))$ where $E$ is the twopomeron 'energy' and $\omega(q)$ is the gluon trajectory, which correspond to free propagation. The diagram in Fig. 1 has to contain only one such factor, which gives an extra factor $E-$ $4 \omega(q)$. The term $E$ transforms into $-\partial / \partial Y$ and the divergent part $4 \omega(q)$ is cancelled in the sum with terms coming from pomeron interactions in Figs. 1 and 2. After this cancellation the infrared finite part of $D^{(1)}$ is given by

$$
D^{(1)}=-\frac{\partial}{\partial Y} \int_{0}^{Y} \mathrm{~d} y^{\prime} \int \frac{\mathrm{d}^{2} q}{(2 \pi)^{2}} P^{2}\left(Y-y^{\prime}, q\right) P^{2}\left(y^{\prime}, q\right) \text {. }
$$




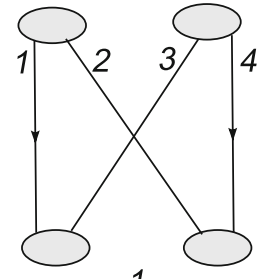

1
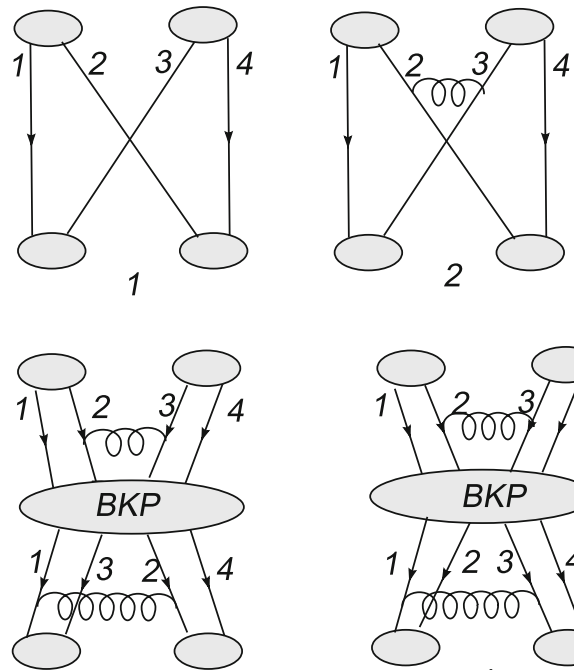

3

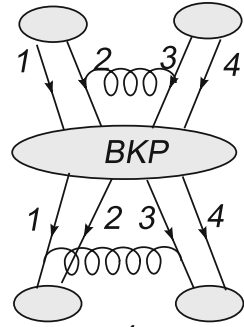

Fig. 1 The scattering amplitude for the collision of two projectiles on two targets

Here $P(y, q)$ is the forward pomeron with rapidity $y$ and relative transversal momentum of reggeised gluons $q$ attached to one of the participant hadrons. It is the solution of the BFKL equation for evolution in rapidity with the initial condition corresponding to two reggeised gluons emitted from the hadron (with their propagators included). In the following, to economise on notation we shall suppress the transversal variables and integrations over them evident from Fig. 1 and rewrite (2) as

$$
\begin{aligned}
D^{(1)}= & -\frac{\partial}{\partial Y} \int_{0}^{Y} \mathrm{~d} y^{\prime} P^{(12)}\left(Y-y^{\prime}\right) P^{(34)}\left(Y-y^{\prime}\right) \\
& \times P^{(13)}\left(y^{\prime}\right) P^{(24)}\left(y^{\prime}\right) .
\end{aligned}
$$

Here we only indicate the numbers of the gluons which combine into pomerons. In this notation the rest contributions $D^{(i)}$ from Fig. 1, $i, i=2,3$, 4 were found to be

$$
\begin{aligned}
D^{(2)}= & \int_{0}^{Y} \mathrm{~d} y^{\prime} P^{(12)}\left(Y-y^{\prime}\right) P^{(34)}\left(Y-y^{\prime}\right)\left(h_{23}+h_{14}\right) P^{(13)} \\
& \times\left(y^{\prime}\right) P^{(24)}\left(y^{\prime}\right) \\
D^{(3)}= & \int_{0}^{Y} \mathrm{~d} y^{\prime} \int_{0}^{y^{\prime}} \mathrm{d} y^{\prime \prime} P^{(12)}\left(Y-y^{\prime}\right) P^{(34)}\left(Y-y^{\prime}\right) \\
& \times \operatorname{AG}\left(y^{\prime}-y^{\prime \prime}\right) B P^{(13)}\left(y^{\prime \prime}\right) P^{(24)}\left(y^{\prime \prime}\right) \\
D^{(4)}= & \int_{0}^{Y} \mathrm{~d} y^{\prime} \int_{0}^{y^{\prime}} \mathrm{d} y^{\prime \prime} P^{(12)}\left(Y-y^{\prime}\right) P^{(34)}\left(Y-y^{\prime}\right) \\
& \times A \tilde{G}\left(y^{\prime}-y^{\prime \prime}\right) A P^{(12)}\left(y^{\prime \prime}\right) P^{(34)}\left(y^{\prime \prime}\right)
\end{aligned}
$$

Here

$h_{i k}=-\omega_{i}-\omega_{k}-v_{i k}$

is the BFKL Hamiltonian for gluons $(i k) ; \omega_{i}$ is the gluon Regge trajectory and $v_{i k}$ is the BFKL interaction between gluons $i$ and $k$ in the symmetric form

$$
\begin{aligned}
v_{i k}\left(q_{i}^{\prime}, q_{k}^{\prime} \mid q_{i}, q_{k}\right)= & 4 \pi \alpha_{s} N_{c} \delta^{2}\left(q_{i}^{\prime}+q_{k}^{\prime}-q_{i}-q_{k}\right) \frac{1}{q_{i}^{\prime} q_{k}^{\prime} q_{i} q_{k}} \\
& \times\left(\frac{q_{i}^{2} q_{k}^{\prime 2}+q_{k}^{2} q_{i}^{\prime 2}}{\left(q_{i}-q_{i}^{\prime}\right)^{2}}-\left(q_{i}+q_{k}\right)^{2}\right) . \quad(8)
\end{aligned}
$$

Combinations of interactions $A$ and $B$ are given by

$A=v_{23}+v_{14}-v_{13}-v_{24}, B=v_{23}+v_{14}-v_{12}-v_{34}$,

both infrared safe. Finally $G$ and $\tilde{G}$ are combinations of the Green functions for the BKP state for different ordering of the four gluons:

$$
\begin{aligned}
G= & \frac{1}{4}\left[G^{1243}+G^{1342}\right], \tilde{G}=\frac{1}{4}\left[G^{1234}+G^{1432}\right. \\
& \left.+G^{1243}+G^{1342}\right]
\end{aligned}
$$

where e.g. $G^{1243}$ satisfies the equation

$$
\left(\frac{\partial}{\partial y}-H_{\mathrm{BKP}}^{1243}\right) G^{1243}(y)=\delta(y)
$$

with the Hamiltonian

$H_{\mathrm{BKP}}^{1243}=-\sum_{i=1}^{4} \omega_{i}-v_{12}-v_{24}-v_{43}-v_{31}$.

In (4)-(6) all quantities between the pomerons are to be understood as operators in the transverse momentum space. Obviously transversal integrations include one, two and three intermediate momenta for (3), (4) and (5) + (6), respectively. Note that in (5) and (6) the interactions in $A$ and $B$ are not to be included into the BKP Green function $G$. The division between them is related to the change in the colour structure accomplished by these interactions. Say in the diagram shown in Figs. 1 and 3 the colour structure above $v_{23}$ and below $v_{12}$ is that of two pomerons and in between is that of the BKP state, that is, of a cylinder with four reggeised gluons on its surface. The interactions $v_{23}$ and $v_{12}$ themselves cannot be included into either of them.

The observed gluons may be emitted (1) both from the same pomeron, (2) from different pomerons, (3) one from a pomeron and the other from the interactions between the exchanged gluons, both explicitly appearing in the diagrams of Fig. 1 and implicit in the BKP state, and finally (4) both from these interactions. Correspondingly we shall study all contributions successively: the contributions from the same or different pomerons in Sect. 2, from a pomeron and explicitly shown interactions in Sect. 3 and both from the interac- 
Fig. 2 'Opening' of the pomeron

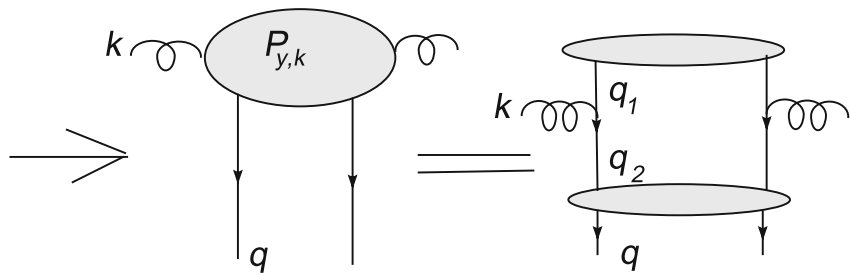

tions and from the BKP state in Sect. 4. Section 5 is devoted to some conclusions.

For the double inclusive cross section in collision of two projectiles with the same momentum $k$ with two projectiles with the same momentum $l$ the high-energy part of the forward scattering amplitude can be presented in the form

$$
\begin{aligned}
H\left(Y, y_{1}, k_{1}, y_{2}, k_{2}\right)= & -i(2 \pi)^{2} \delta\left(\kappa_{+}\right) \delta\left(q_{-}\right) N_{c}^{2}(k l)^{2} \\
& \times F\left(Y, y_{1}, k_{1}, y_{2}, k_{2}\right) .
\end{aligned}
$$

Here $Y$ is the overall rapidity, $\kappa$ and $q$ are the momenta transferred to the projectile nucleus and target nucleus respectively with $\kappa_{-}=\kappa_{\perp}=q_{+}=q_{\perp}=0$. Factor $N_{c}^{2}(k l)^{2}$ is present in all diagrams, so that it is convenient to separate it. $F$ gives the contribution from the diagram for the $S$-matrix (hence $-i$ in (13)). Arguments $y_{1}, k_{1}$ and $y_{2}, k_{2}$ are the rapidities and transverse momenta of the observed gluons. We assume $y_{1}>>y_{2}$ motivated by the desire to study long-range correlations in rapidity (in practice $y_{1}-y_{2} \sim 2 \div 3$ ). The double inclusive cross section for two-nucleon-two-nucleon interaction in nucleus-nucleus scattering at a given impact parameter $b$ is then

$$
\begin{aligned}
& I_{A B}\left(Y, b, y_{1}, k_{1}, y_{2}, k_{2}\right) \equiv \frac{(2 \pi)^{2} \mathrm{~d} \sigma_{A B}}{\mathrm{~d}^{2} b \mathrm{~d} y_{1} \mathrm{~d}^{2} k_{1} \mathrm{~d} y_{2} \mathrm{~d}^{2} k_{2}} \\
& =\frac{1}{4} A(A-1) B(B-1) T_{A B}^{(2)}(b) F\left(Y, y_{1}, k_{1}, y_{2}, k_{2}\right),
\end{aligned}
$$

where the transverse density for two pairs of participants is

$T_{A B}^{(2)}(b)=\int \mathrm{d}^{2} b_{A} \mathrm{~d}^{2} b_{B} T_{A}^{2}\left(b_{A}\right) T_{B}^{2}\left(b_{B}\right) \delta^{2}\left(b_{A}-b_{B}-b\right)$

and $T_{A, B}(b)$ are the nuclear profile functions normalised to unity. For deuteron-deuteron scattering we find instead

$I_{\mathrm{dd}}\left(Y, y_{1}, k_{1}, y_{2}, k_{2}\right)=\frac{1}{4}\left\langle\frac{1}{2 \pi r^{2}}\right\rangle_{d}^{2} F(Y, y, k)$.

In the following we sometimes suppress the arguments $Y, y_{1}, k_{1}, y_{2}, k_{2}$ in our formulae.

After separation of the $\delta$-functions in (13) the corresponding diagrams contain internal longitudinal integrations over intermediate gluon momenta. For each of the observed intermediate gluon these integrations are included in the definition of $I$ leaving only a factor $1 / 4 \pi$. Other interactions may refer to either real gluons in the intermediate state or virtual gluons inside the production amplitudes. If the gluon is real, longitudinal integrations reduce to the integration over its rapidity with the same factor $1 / 4 \pi$. If the gluon is virtual, integration of its propagator lifts one of the integrations leaving the integration over its rapidity with an additional factor $-i / 4 \pi$ (see [1]). Taking into account that inclusion of the virtual gluon provides an additional factor $(-i)^{3}$ we find that in the end virtual integrations give the same result as real ones. This is important for our calculations: the contribution from the internal gluon line does not depend on whether it refers to the real gluon (is 'cut') or the virtual one (is 'uncut'). Apart from integrations over the unobserved or virtual gluon rapidities, function $F$ is just a contribution from the diagrams with transverse integrations over the gluon momenta in accordance with the conservation laws.

\section{Contribution from the pomerons}

For the following note that in our kinematics the pomeron wave function is real. The inclusive cross section for the production of a gluon with rapidity $y$ and transverse momentum $k$ from the pomeron is well known. It corresponds to substitution of the pomeron $P\left(Y-y^{\prime}, q\right)$ by the inclusive cross section $P_{y, k}\left(Y, y^{\prime}, q\right)$ obtained by 'opening' one of the BFKL interactions inside as shown in Fig. 2.

In our shorthand notation, suppressing the evident transversal integrations, we have

$P_{y, k}\left(Y, y^{\prime}\right)=P(Y-y) v G_{(}\left(y-y^{\prime}\right)$,

Likewise the double inclusive cross section corresponds to substitution of the pomeron $P\left(Y-y^{\prime}, q\right)$ by the double inclusive cross section $P_{y_{1}, k_{1}, y_{2}, k_{2}}\left(Y, y^{\prime}, q\right)$ illustrated in Fig. 3 and given by

$$
P_{y_{1}, k_{1}, y_{2}, k_{2}}\left(Y, y^{\prime}\right)=P\left(Y-y_{1}\right) v G\left(y_{1}-y_{2}\right) v G\left(y_{2}-y^{\prime}\right) .
$$

Note that we assume $y_{1}>y_{2}$, so that diagrams with the two observed gluons emitted from the same (vertical) reggeon lines should be summed with those which contain unobserved gluons emitted at intermediate rapidities, which converts these lines into the full BFKL Green function.

The total double inclusive cross section from the pomerons can be separated into two parts: with both gluons emitted from the same pomeron and with gluons emitted from dif- 
Fig. 3 Double 'opening' of the pomeron
Fig. 4 Cuts for emission of two gluons from different pomerons
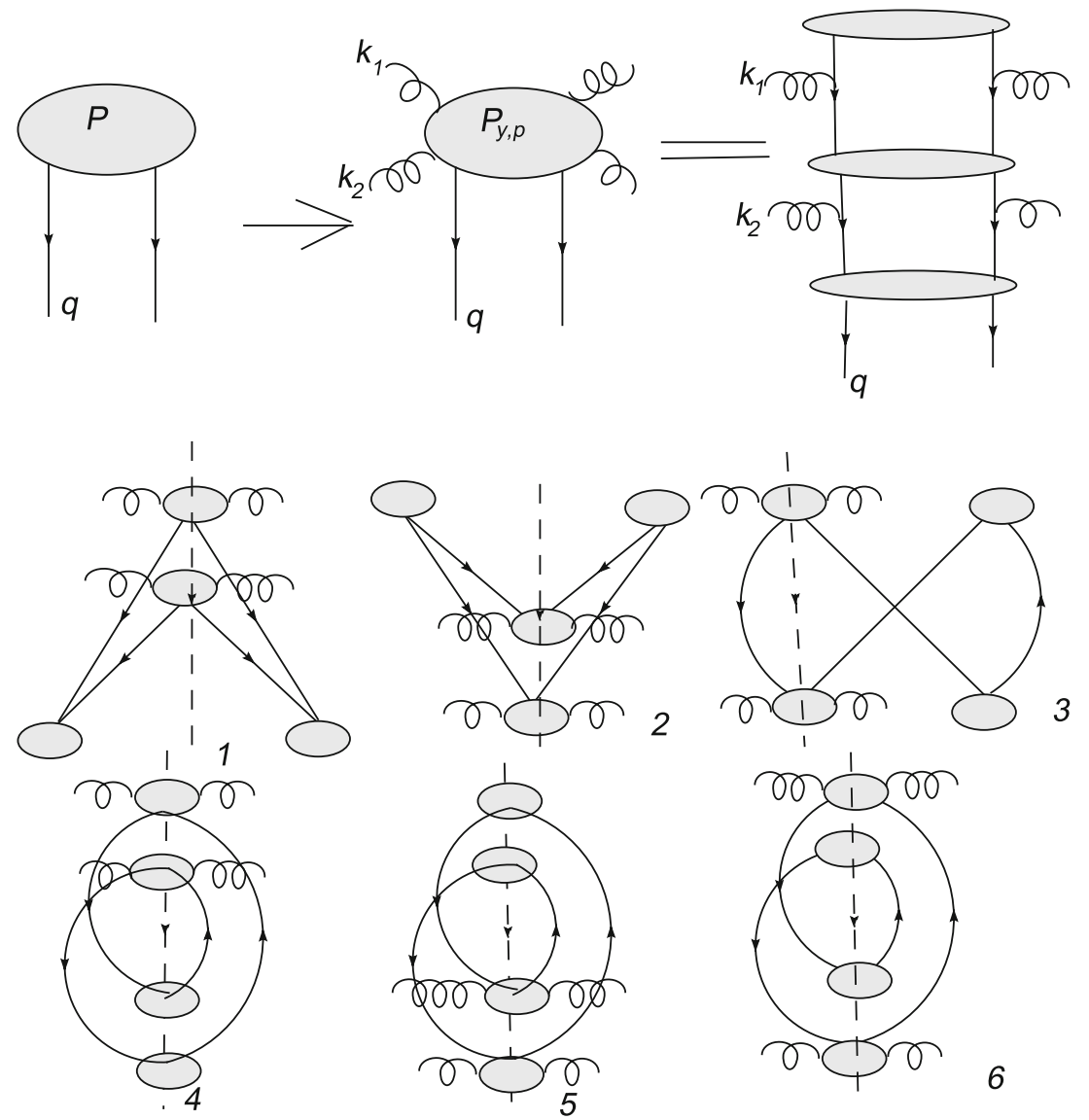

ferent pomerons from among the four which are present in the diagrams of Fig. 1.

In the first case the double inclusive cross section will be given by the same formulae as for the single inclusive cross section in [2] in which one only has to substitute

$P_{y, k}(Y, q) \rightarrow P_{y_{1}, k_{1}, y_{2}, k_{2}}(Y, q)$.

So one finds the contribution to $F$

$F^{(1)}=2 D\left(\right.$ one of $\left.P^{\prime} s \rightarrow P_{y_{1}, k_{1}, y_{2}, k_{2}}\right)$

where $D$ is given by (3)-(6) and the extra factor 2 is due to two possibilities of choosing the production amplitude and its complex conjugate.

So we study in some detail only the second case when the two observed gluons belong to different pomerons. In this case the double inclusive cross section will be obtained if we cut the diagrams in all possible ways and substitute two of the cut pomerons according to (17). A particular cut may also pass or not pass through explicit interactions in Fig. 1-2-4 or inside the BKP state. The resulting cross section from the pomerons will not depend on whether these extra interactions are cut or not, since the cut BFKL interaction is equal to uncut one. So one can study different contributions from the pomerons forgetting about these extra interactions. The relevant cuts in this case are shown in Fig. 4.
Diagrams 1 and 2 correspond to diffractive configurations respective to the target (DT) or projectile (DP). Diagram 3 illustrates the single cut configuration $(\mathrm{S})$ in which one projectile and one target are cut. Diagrams 4-6 show the double cut configuration (DC) in which both projectiles and both targets are cut. Note that if the two gluons are both emitted from the pomerons attached to the projectile (Fig. 4-1) or both emitted from the pomerons attached to the target (Fig. 4-2) they can bear any rapidity $y_{1}$ or $y_{2}$. If one of the gluons is emitted from the pomeron attached to the projectile and the other from the pomeron attached to the target then the first should have rapidity $y_{1}$ and the second rapidity $y_{2}$. So effectively the contributions from Fig. 4-1, 2, 4 and 5 are to be multiplied by 2 .

We start with the diffractive contributions. The one respective to the target (Fig. 4-1) gives the contribution to the highenergy part $F$ from Fig. 1-1:

$F_{\text {DT }}^{2,1}=4 \frac{\partial}{\partial Y} \int_{0}^{Y} \mathrm{~d} y^{\prime} P_{y_{1}, k_{1}}^{(12)}\left(Y, y^{\prime}\right) P_{y_{2}, k_{2}}^{(34)}\left(Y, y^{\prime}\right) P^{(13)}\left(y^{\prime}\right) P^{(14)}$.

The coefficient 4 takes into account two projectiles and two targets. The diffractive contribution respective to the projec- 
tile (Fig. 4-2) gives

$$
\begin{aligned}
F_{\mathrm{DP}}^{2,1}= & 4 \frac{\partial}{\partial Y} \int_{0}^{Y} \mathrm{~d} y^{\prime} P^{(12)}\left(Y-y^{\prime}\right) P^{(34)}\left(Y-y^{\prime}\right) \\
& \times P_{y_{1}, k_{1}}^{(13)}\left(y^{\prime}, 0\right) P_{y_{2}, k_{2}}^{(24)}\left(y^{\prime}, 0\right) .
\end{aligned}
$$

The single cut contributions (Fig. 4-3) enter with the minus sign. In fact they contain one exchanged reggeon on the left and three on the right giving $i(-i)^{3}=-1$. Their contribution is

$$
\begin{aligned}
F_{S}^{2,1}= & -4 \frac{\partial}{\partial Y} \int_{0}^{Y} d y^{\prime} P_{y_{1}, k_{1}}^{(12)}\left(Y, y^{\prime}\right) P^{(34)}\left(Y-y^{\prime}\right) \\
& \times P_{y_{2}, k_{2}}^{(13)}\left(y^{\prime}, 0\right) P^{(24)}\left(y^{\prime}\right) .
\end{aligned}
$$

The coefficient 4 again takes into account interchanges of the two projectiles and two targets.

Finally the DC contribution (Figs. 4-4, 5 and 6) gives

$$
\begin{aligned}
F_{\mathrm{DC}}^{2,1}= & 4 \frac{\partial}{\partial Y} \int_{0}^{Y} \mathrm{~d} y^{\prime}\left(P_{y_{1}, k_{1}}^{(12)}\left(Y, y^{\prime}\right) P_{y_{2}, k_{2}}^{(34)}\left(Y, y^{\prime}\right) P^{(13)}\left(y^{\prime}\right)\right. \\
& \times P^{(24)}\left(y^{\prime}\right)+P^{(12)}\left(Y-y^{\prime}\right) P^{(34)}\left(Y-y^{\prime}\right) \\
& \times P_{y_{1}, k_{1}}^{(13)}\left(y^{\prime}, 0\right) P_{y_{2}, k_{2}}^{(24)}\left(y, 0^{\prime}\right)+2 P_{y_{1}, k_{1}}^{(12)}\left(Y, y^{\prime}\right) \\
& \left.\times P^{(34)}\left(y-y^{\prime}\right) P_{y_{2}, k_{2}}^{(13)}\left(y^{\prime}, 0\right) P^{(24)}\left(y^{\prime}\right)\right) .
\end{aligned}
$$

In the first two terms the numerical factor is 4 , corresponding to different choice of $\left(y_{1}, k_{1}\right)$ and $\left(y_{2}, k_{2}\right)$ in upper or lower pomerons and two and two different diagrams for the DC configuration. In the last term this factor is twice larger since $\left(y_{1}, k_{1}\right)$ and $\left(y_{2}, k_{2}\right)$ can be distributed in four manners between upper and lower pomerons.

In the sum the $S$ contribution cancels half of the last term in the DC contributions and we get the final $F$ from the gluons inside the pomerons as

$$
\begin{aligned}
F^{2,1}= & 4 \frac{\partial}{\partial Y} \int_{0}^{Y} \mathrm{~d} y^{\prime}\left(2 P_{y_{1}, k_{1}}^{(12)}\left(Y, y^{\prime}\right) P_{y_{2}, k_{2}}^{(34)}\left(Y, y^{\prime}\right) P^{(13)}\left(y^{\prime}\right)\right. \\
& \times P^{(24)}\left(y^{\prime}\right)+2 P^{(12)}\left(Y-y^{\prime}\right) P^{(34)}\left(Y-y^{\prime}\right) \\
& \times P_{y_{1}, k_{1}}^{(13)}\left(y^{\prime}, 0\right) P_{y_{2}, k_{2}}^{(24)}\left(y, 0^{\prime}\right)+P_{y_{1}, k_{1}}^{(12)}\left(Y, y^{\prime}\right) \\
& \left.\times P^{(34)}\left(y-y^{\prime}\right) P_{y_{2}, k_{2}}^{(13)}\left(y^{\prime}, 0\right) P^{(24)}\left(y^{\prime}, q\right)\right)
\end{aligned}
$$

As mentioned, inclusion of other interactions in between does not change the form of the result, which is obtained from the formulae for the forward amplitude making the substitutions (17) in the same manner as above. Referring the reader to [2] for the details we only present the final results here. For the diagram in Fig. 1-2 we find the corresponding $F$ as

$$
\begin{aligned}
F^{2,2}= & 4 \int_{0}^{Y} \mathrm{~d} y^{\prime}\left(2 P_{y_{1}, k_{1}}^{(12)}\left(Y, y^{\prime}\right) P_{y_{2}, k_{2}}^{(34)}\left(Y, y^{\prime}, q^{\prime}\right) H P^{(13)}\left(y^{\prime}\right)\right. \\
& \times P^{(24)}\left(y^{\prime}\right)+2 P^{(12)}\left(Y-y^{\prime}\right) P^{(34)}\left(Y-y^{\prime}\right) \\
& \times H P_{y_{1}, k_{1}}^{(13)}\left(y^{\prime}, 0\right) P_{y_{2}, k_{2}}^{(24)}\left(y, 0^{\prime}\right)+P_{y_{1}, k_{1}}^{(12)}\left(Y, y^{\prime}\right) \\
& \times P^{(34)}\left(y-y^{\prime}\right) H P_{y_{2}, k_{2}}^{(13)}\left(y^{\prime}, 0, q^{\prime}\right) P^{(24)}\left(y^{\prime}\right) \\
& \left.+P_{y_{1}, k_{1}}^{(12)}\left(Y, y^{\prime}\right) P^{(34)}\left(y-y^{\prime}\right) H P^{(13)}\left(y^{\prime}\right) P_{y_{2}, k_{2}}^{(24)}\left(y^{\prime}, 0\right)\right) .
\end{aligned}
$$

For the diagram in Fig. 1-3 with the BKP state we have the amplitude $F$ :

$$
\begin{aligned}
F^{2,3}= & 4 \int_{0}^{Y} \mathrm{~d} y^{\prime} \int_{0}^{y^{\prime}} \mathrm{d} y^{\prime \prime}\left(2 P_{y_{1}, k_{1}}^{(12)}\left(Y, y^{\prime}\right) P_{y_{2}, k_{2}}^{(34)}\right. \\
& \times\left(Y, y^{\prime}, q_{4}\right) M\left(y^{\prime}-y^{\prime \prime}\right) P^{(13)}\left(y^{\prime \prime}\right) P^{(24)}\left(y^{\prime \prime}\right) \\
& +2 P^{(12)}\left(Y-y^{\prime}\right) P^{(34)}\left(Y-y^{\prime}\right) M\left(y^{\prime}-y^{\prime \prime}\right) \\
& \times P_{y_{1}, k_{1}}^{(13)}\left(y^{\prime \prime}, 0\right) P_{y_{2}, k_{2}}^{(24)}\left(y^{\prime \prime}\right)+P_{y_{1}, k_{1}}^{(12)}\left(Y, y^{\prime}\right) \\
& \times P^{(34)}\left(Y-y^{\prime}\right) M\left(y^{\prime}-y^{\prime \prime}\right) P_{y_{2}, k_{2}}^{(13)}\left(y^{\prime \prime}, 0\right) P^{(24)}\left(y^{\prime \prime}\right) \\
& +P_{y_{1}, k_{1}}^{(12)}\left(Y, y^{\prime}\right) P^{(34)}\left(Y-y^{\prime}\right) M\left(y^{\prime}-y^{\prime \prime}\right) \\
& \left.\times P^{(13)}\left(y^{\prime \prime}\right) P_{y_{2}, k_{2}}^{(24)}\left(y^{\prime \prime}, 0\right)\right) .
\end{aligned}
$$

Here $M=\operatorname{AG}\left(y^{\prime}-y^{\prime \prime}\right) B$ where $A, B$ and $G$ are defined in (9) and (10)

The contribution $F^{2,4}$ from the diagram in Fig. 1-4 will be given by a similar expression with the lower pomerons $P^{(13)} P^{(24)} \rightarrow P^{(12)} P^{(34)}$ and $M \rightarrow \tilde{M}=A \tilde{G} A$. The total contribution of emission from two different pomerons is

$$
F^{(2)}=\sum_{i=1}^{4} F^{2, i} \text {. }
$$

\section{One gluon emitted from the pomeron, the other from interactions}

\subsection{Single interaction between the pomerons}

Here we study the contribution to the double inclusive cross section in which one gluon comes from the four pomerons attached to the projectiles or targets and the other from opening the interaction in the diagram of Fig. 1-2. Again we shall have different contributions depending on the cuts in the overall amplitude. Some typical ones are shown in Fig. 5-1-3 (the full list of diagrams may be inferred from those in [2]).

Diagrams 1 and 2 describe the two D configurations, DT and DP, diagram 3 describes the $\mathrm{S}$ configuration. Note that the DC contribution does not contain any observed gluon in the intermediate state and so gives no contribution. All contributions should be taken with coefficient 4 due to interchanges 

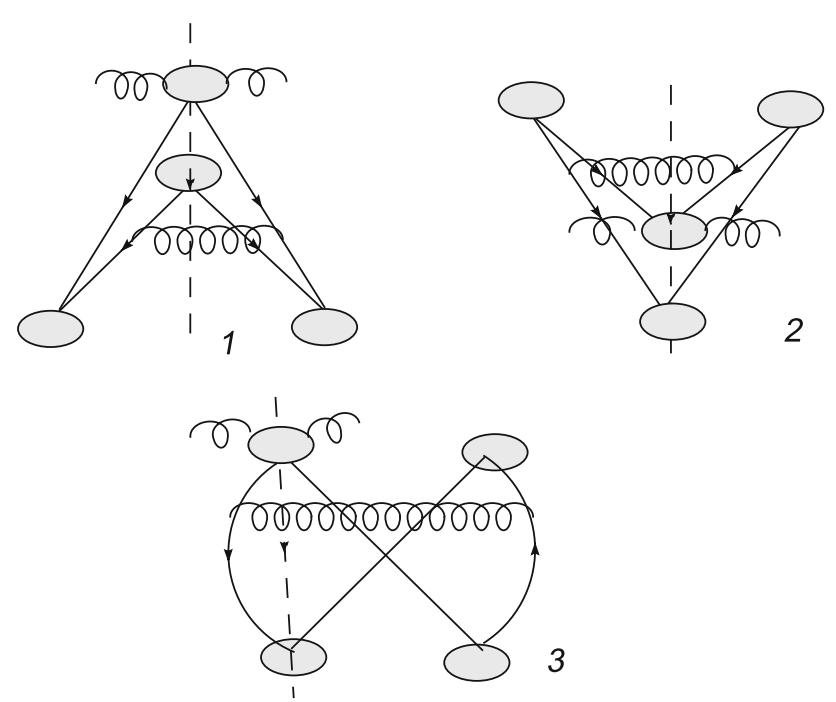

Fig. 5 Some typical diagrams for emission of one gluon from the pomeron and another from interaction, with diffractive cuts $(1,2)$ and a single cut (3). The rapidity and momentum of the interaction are assumed to be fixed either to $\left(y_{1}, k_{1}\right)$ or $\left(y_{2}, k_{2}\right)$

of projectiles and targets, which become non-equivalent due to attachment of cut or non-cut pomerons, Also in the diagrams Figs. 5-1 and 3 the gluon emitted from the pomeron is to have the larger rapidity than the one emitted from the interaction and in the diagram Fig. 5-2 and the one similar to Fig. 5-3 with emission from the lower pomeron the gluon emitted from the pomeron is to have the lower rapidity. Taking into account contributions from all diagrams we find that the $S$ contributions cancel $1 / 2$ of the diffractive contributions. (The extra factor in the $\mathrm{S}$ contribution appears because of the fact that of two possible interactions $v_{23}$ and $v_{13}$ only half of them contains a real gluon in the intermediate state.) So for the case when the gluon of higher rapidity is emitted from the pomeron the result is

$$
\begin{aligned}
F^{3, h}= & 4 \int_{0}^{Y} \mathrm{~d} y^{\prime} P_{y_{1}, k_{1}}^{(12)}\left(Y, y^{\prime}\right) P^{(34)}\left(Y-y^{\prime}\right) \\
& \times v_{23} P^{(13)}\left(y^{\prime}\right) P^{(24)}\left(y^{\prime}\right)
\end{aligned}
$$

and for the case when the gluon of higher rapidity is emitted from the interaction the result is

$$
\begin{aligned}
F^{3, l}= & 4 \int_{0}^{Y} \mathrm{~d} y^{\prime} P^{(12)}\left(Y-y^{\prime}\right) P^{(34)}\left(Y-y^{\prime}\right) v_{23} P_{y_{2}, k_{2}}^{(13)} \\
& \times\left(y^{\prime}, 0\right) P^{(24)}\left(y^{\prime}\right) .
\end{aligned}
$$

The total is

$$
F^{(3)}=F^{3, h}+F^{3, l} \text {. }
$$
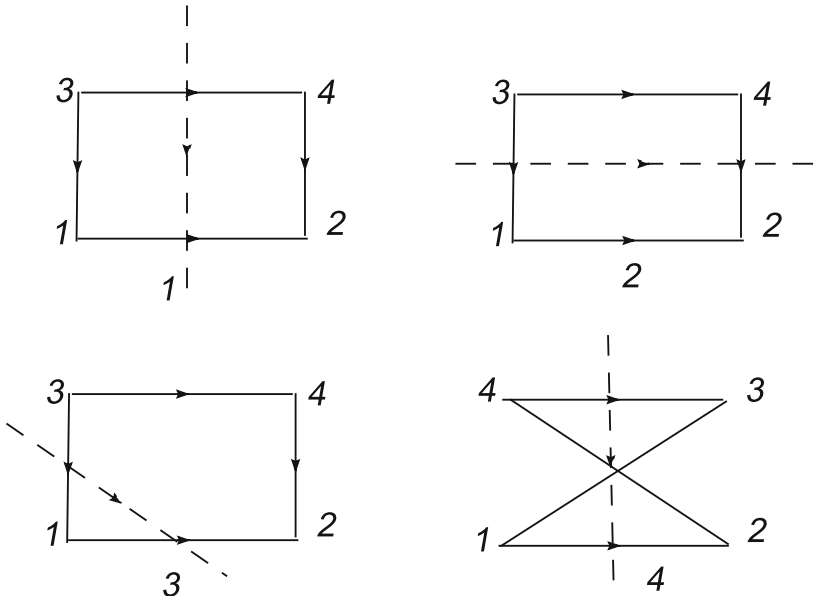

Fig. 6 Cut interactions between different exchanged gluons for different configurations

\subsection{Two interactions between the pomerons} with redistribution of colour

In this subsection we study contributions to the double inclusive cross section which comes from opening one of the pomerons and one of the interactions explicitly shown in Fig. 1-3. The relevant cuts are to pass through the interaction which contains the observed gluon. They have also to pass through the diagram as a whole and thus have to pass through some pomerons attached to the projectile and target and also through the BKP state. As a diagram the latter is a cylinder with four reggeised gluons arranged on its surface in the order 1243 or 1342 with interaction between neighbours. The cut of the amplitude generates a cutting plane between reggeised gluons in the BKP state. As discussed in [2], the position of this plane is totally determined by the cut passing through the pomerons and for different configurations is shown in Fig. 6 by its trace on the plane orthogonal to the cylinder.

The forward scattering amplitude is given by (5). Since cut and uncut interactions give the same contribution, this also means that in all cases the BKP state will appear as a whole between the interactions which generate the observed gluon. So to simplify notations in some of the following formulae we put $G(y) \rightarrow 1$ and introduce this Green function between the interactions only in the end.

The double inclusive cross sections correspond to opening one of the pomerons and one of the interactions. Some typical diagrams illustrating contributions from various configurations are shown in Fig. 7 (where we suppressed $G$ between the interactions).

In the double inclusive cross section with one of the gluons emitted from the upper pomerons at rapidity $y_{1}$ we are to open either the upper interactions, which implies fixing $Y-$ $y^{\prime}=y_{2}$ and the momenta transferred to $k_{2}$ in one of the four 


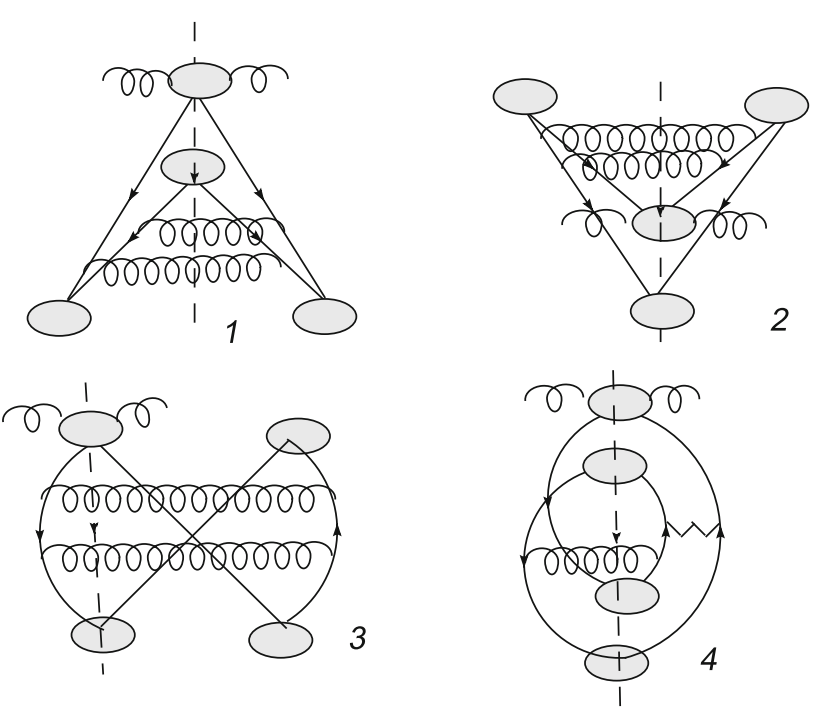

Fig. 7 Typical diagrams for emission of one gluon from the pomeron and another from one of the two interactions with redistribution of colour in the diffractive $(1,2)$, single cut (3) and double cut (4) configurations. The BKP state between the interactions is not shown

interactions on the left in (5), or the lower interactions, which implies fixing $y^{\prime \prime}=y_{2}$ and the momentum transferred in one of the interactions on the right in (5).

In both cases contributions will come from DT, DP, S and DC configurations. We shall write our results for the highenergy part $F$ with one of the gluon emitted from the upper pomerons in the following form. For emission of the second gluon from the interaction higher in rapidity

$$
\begin{aligned}
F^{4, h h}= & \int_{0}^{y^{\prime}} \mathrm{d} y^{\prime \prime} P_{y_{1}, k_{1}}^{(12)}\left(Y, y^{\prime}\right) P^{(34)}\left(Y-y^{\prime}\right) \\
& \times f_{\text {high }}\left(y^{\prime}-y^{\prime \prime}\right) P^{(13)}\left(y^{\prime \prime}\right) P^{(24)}\left(y^{\prime \prime}\right), y^{\prime}=y_{2}
\end{aligned}
$$

and for emission of the second gluon from the interaction lower in rapidity

$$
\begin{aligned}
F^{4, h l}= & \int_{y^{\prime \prime}}^{Y} \mathrm{~d} y^{\prime} P_{y_{1}, k_{1}}^{(12)}\left(Y, y^{\prime}\right) P^{(34)}\left(Y-y^{\prime}\right) \\
& \times f_{\text {low }}\left(y^{\prime}-y^{\prime \prime}\right) P^{(13)}\left(y^{\prime \prime}\right) P^{(24)}\left(y^{\prime \prime}\right), y^{\prime \prime}=y_{2}
\end{aligned}
$$

The operators $f_{\text {high }}$ and $f_{\text {low }}$ are different for different configurations. A derivation of $f_{\text {high }}$ and $f_{\text {low }}$ can be found in [2]. One has

$$
f_{\text {high }}=\operatorname{AG}\left(y^{\prime}-y^{\prime \prime}\right)\left(v_{23}-v_{34}\right)=\frac{1}{2} \operatorname{AG}\left(y^{\prime}-y^{\prime \prime}\right) B
$$

with the observed gluon emitted from the interaction on the left. Remarkably $f_{\text {low }}$ is given by the same formula but with the gluon emitted from the interaction on the right.

A similar contribution has to be added in the case when the gluon of a higher rapidity is emitted from one of the interactions and the one of lower rapidity from one of the lower pomerons. This contribution is given by two terms similar to (32) and (33): for emission of the first gluon from the interaction higher in rapidity

$$
\begin{aligned}
F^{4, l h}= & \int_{0}^{y^{\prime}} \mathrm{d} y^{\prime \prime} P^{(12)}\left(Y-y^{\prime}\right) P^{(34)}\left(Y-y^{\prime}\right) f_{\mathrm{high}}\left(y^{\prime}-y^{\prime \prime}\right) \\
& \times P_{y_{2}, k_{2}}^{(13)}\left(y^{\prime \prime}, 0\right) P^{(24)}\left(y^{\prime \prime}\right), y^{\prime}=y_{1},
\end{aligned}
$$

and for emission of the first gluon from the interaction lower in rapidity

$$
\begin{aligned}
F_{l l}^{4, l l}= & \int_{y^{\prime \prime}}^{Y} \mathrm{~d} y^{\prime} P^{(12)}\left(Y-y^{\prime}\right) P^{(34)}\left(Y-y^{\prime}\right) f_{l o w}\left(y^{\prime}-y^{\prime \prime}\right) \\
& \times P_{y_{2}, k_{2}}^{(13)}\left(y_{1}\right) P_{24}\left(y_{1}\right), y^{\prime \prime}=y_{1},
\end{aligned}
$$

with the same $f_{\text {high }}$ and $f_{\text {low }}$ as given before.

Our final expression for the emission of one of the gluons from the two explicit interactions in the diagram with the other emitted from one of the pomerons is therefore given by the sum

$F^{(4)}=F^{4, h h}+F^{4, h l}+F^{4, l h}+F^{4, l l}$

with the common function

$f\left(y^{\prime}-y^{\prime \prime}\right)=\frac{1}{2} \operatorname{AG}\left(y^{\prime}-y^{\prime \prime}\right) B$.

The observed gluon may be located either in the left interaction or the right one and correspondingly $y^{\prime}=y_{2}$ or $y^{\prime \prime}=y_{2}$ when the first gluon is emitted from the upper pomeron or $y^{\prime}=y_{1}$ or $y^{\prime \prime}=y_{1}$ when the second pomeron is emitted from the lower pomeron.

\subsection{Two interactions between the pomerons with direct colour transmission (DCT)}

The amplitude itself is given by (6) and shown in Fig. 1-4. In this case the identity of the two projectiles and two targets generates symmetries in independent interchanges $1 \leftrightarrow 2$ or $3 \leftrightarrow 4$.

As in the previous subsection the relevant cuts are to pass through the interaction which contains the observed gluon and also through the diagram as a whole and thus through the BKP state. The position of the latter cut will again be totally determined by the cut passing through the pomerons, and is shown Fig. 8 for different configurations.

Again, since cut and uncut interactions give the same contribution, in all cases the BKP state will appear as a whole between the interactions which generate the observed gluon and we can write contributions from the explicitly shown interactions formally putting $\tilde{G} \rightarrow 1$ and afterwards introduce this Green functions between the interactions. Some 


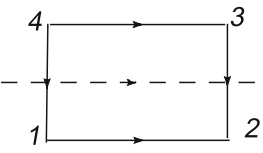

$D D$
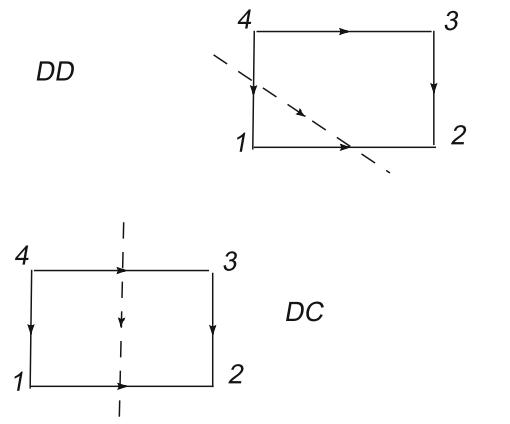

Fig. 8 Cut interactions between the different gluons for different cut configurations with direct colour transmission

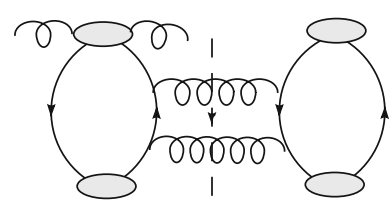

1

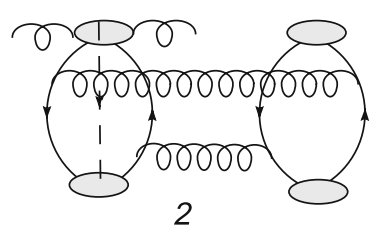

2

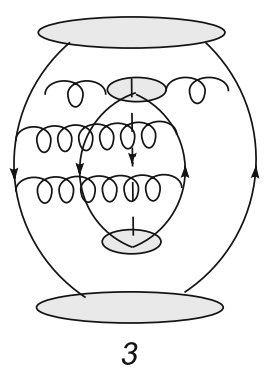

Fig. 9 Typical diagrams for emission of one gluon from the pomeron and another from one of the two interactions with direct colour transmission in the double diffractive (1), single cut (2) and double cut (3) configurations. The BKP state between the interactions is not shown

typical diagrams for the double inclusive cross sections corresponding to opening one of the pomerons and one of the interactions are shown in Fig. 9.

As in the previous subsection the inclusive cross section can be separated into those in which the gluon of higher rapidity $y_{1}$ is emitted from the upper pomeron and those in which the gluon of lower rapidity $y_{2}$ is emitted from the lower pomeron. In each case the other gluon may be emitted from one of the interactions. So in the first case one has to fix in the upper(lower) interaction $Y-y^{\prime}=y_{2}\left(y^{\prime}=y_{2}\right)$ and the transferred momenta to $k_{2}$ and in the second case fix in the upper lower) interaction $Y-y^{\prime}=y_{1}\left(y^{\prime}=y_{1}\right)$ and the transferred momentum to $k_{1}$. In both cases we write out results for the corresponding high-energy part $F^{(5)}$ in the same forms as before, (32), (33), (35) and (36), in which we only have to change the lower pomerons $P^{(13)} P^{(24)} \rightarrow$ $P^{(12)} P^{(34)}$.

The derivation of the new $f_{\text {high }}$ and $f_{\text {low }}$ can be found in [2]. It turns out that both are again given by the same formula $f=A \tilde{G}\left(y^{\prime}-y^{\prime \prime}\right) A$, where the gluon of higher rapidity is emitted from the left interactions and the one of lower rapidity from the right interaction. The total inclusive function $F^{(5)}$ will be given by a sum similar to (37):

$F^{(5)}=F^{5, h h}+F^{5, h l}+F^{5, l h}+F^{5, l l}$

\subsection{The BKP state}

The simple inclusive cross section from the BKP state was derived in [2]. So all we have to do is combine it with the inclusive cross section from the pomeron. With the colour redistribution the cross section is illustrated in Fig. 10. Only some typical diagrams are shown in the diffractive, single and double cut configurations. The full list of diagrams as before can be inferred from [2].

We present the results in the form similar to the ones before. For the case with colour redistribution and the gluon $\left(y_{1}, k_{1}\right)$ emitted from the upper pomeron

$$
\begin{aligned}
F^{6, h}= & \int_{0}^{Y} \mathrm{~d} y^{\prime} \int_{0}^{y^{\prime}} \mathrm{d} y^{\prime \prime} P_{y_{1}, k_{1}}^{(12)}\left(Y, y^{\prime}\right) P^{(34)}\left(Y-y^{\prime}\right) \mathrm{AG}_{y_{2}, k_{2}}^{1243} \\
& \times\left(\left\{v_{\mathrm{BKP}}^{1243}\right\}, y^{\prime}, y^{\prime \prime}\right) B P^{(13)}\left(y^{\prime \prime}\right) P^{(24)}\left(y^{\prime \prime}\right) .
\end{aligned}
$$

For the same case and the gluon $\left(y_{2}, k_{2}\right)$ emitted from the lower pomeron

$$
\begin{aligned}
F^{6, l}= & \int_{0}^{Y} \mathrm{~d} y^{\prime} \int_{0}^{y^{\prime}} \mathrm{d} y^{\prime \prime} P^{(12)}\left(Y-y^{\prime}\right) P^{(34)}\left(Y-y^{\prime}\right) A G_{y_{1}, k_{1}}^{1243} \\
& \times\left(\left\{v_{\mathrm{BKP}}^{1243}\right\}, y^{\prime}, y^{\prime \prime}\right) B P_{y_{2} k_{2}}^{(13)}\left(y^{\prime \prime}, 0\right) P^{(24)}\left(y^{\prime \prime}\right)
\end{aligned}
$$

Recall that $A$ and $B$ are defined by (9), $v_{\mathrm{BKP}}^{1243}=v_{12}+v_{24}+$ $v_{43}+v_{31}$ is the sum of interactions between the neighbouring gluons in order 1243 and $G_{y, k}^{1243}\left(\{f\}, y^{\prime}, y^{\prime \prime}\right)$ is given symbolically as

$G_{y, k}^{1243}\left(\{f\}, y^{\prime}, y^{\prime \prime}\right)=G^{1243}\left(y^{\prime}-y\right) f G^{1243}\left(y-y^{\prime \prime}\right)$,

where $f$ corresponds to emission of a gluon $(y, k)$. The total contribution is

$F^{(6)}=F^{6, h}+F^{6, l}$.

For the case without colour redistribution to obtain the corresponding contribution $F^{(7)}$ one has to change

$$
\begin{aligned}
& P^{(13)} P^{(24)} \rightarrow P^{(12)} P^{(34)} \text { and } \\
& \quad \mathrm{AG}_{y_{1}, k_{1}}^{1243}\left(\left\{v_{\mathrm{BKP}}^{1243}\right\}, y^{\prime}, y^{\prime \prime}\right) B \rightarrow A\left[G_{y_{1}, k_{1}}^{1234}\left(\left\{v_{\mathrm{BKP}}^{1234}\right\}, y^{\prime}, y^{\prime \prime}\right)\right. \\
& \left.\quad+G_{y_{1}, k_{1}}^{1243}\left(\left\{v_{\mathrm{BKP}}^{1243}\right\}, y^{\prime}, y^{\prime \prime}\right)\right] A .
\end{aligned}
$$


Fig. 10 Gluon production from the pomeron and the BKP state in the diffractive (1), single (2) and double (2) cut configurations

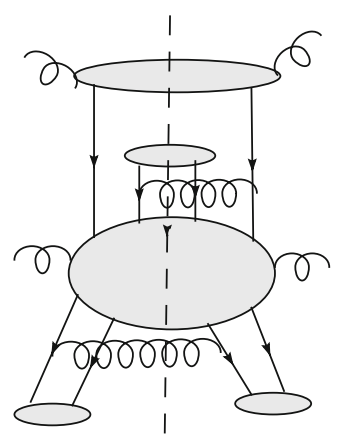

1

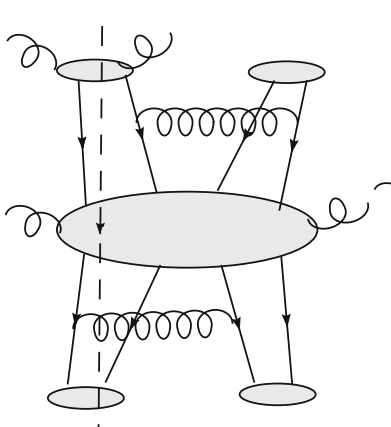

2

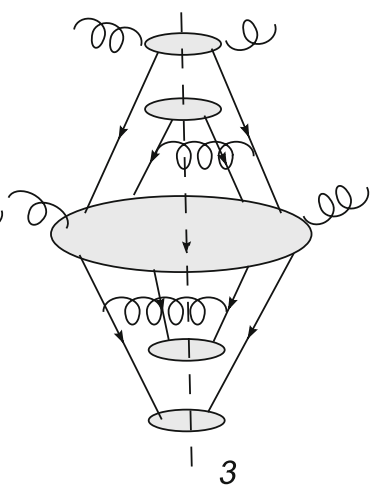

\section{Both gluons emitted from the interaction}

\subsection{Two interactions with redistribution of colour}

Here we study the contribution in which one gluon is emitted from the upper interaction in Fig. 1-3 and the other from the lower interaction. As before the cut BKP blob will enter as a whole and we can formally put $G \rightarrow 1$ restoring $G$ in the final formulae. The diagrams illustrating the double inclusive cross section will be the same as in Fig. 7 in which one should suppress emission from the pomeron but assume instead that both interactions are opened, that is, with fixed rapidities and transverse momenta. The amplitude $F$ in this case can be written as

$F^{(8)}=P^{(12)}\left(Y-y_{1}\right) P^{(34}\left(Y-y_{1}\right) f_{k_{1}, k_{2}}^{(8)} P^{(13)}\left(y_{2}\right) P^{(24)}\left(y_{2}\right)$,

where $f^{(8)}\left(k_{1}, k_{2}\right)$ is in fact made of products of upper and lower interactions in which the transferred momenta are fixed at $k_{1}$ for the upper interaction and $k_{2}$ for the lower one. The final form of $f$ is determined after the study of D,S and DC configurations. As compared to the previous Sect. 3.1 we have to retain only diagrams with two gluons in the intermediate state.

From the D configurations with respect to the target and projectile we find

$f_{\mathrm{DT}}=2\left(v_{23}+v_{14}\right) B, f_{\mathrm{DP}}=2 A\left(v_{23}+v_{14}\right)$.

From the $\mathrm{S}$ configuration

$f_{S}=-A B-\left(v_{23}-v_{14}\right)\left(v_{23}-v_{14}\right)$.

The DC contributions with two gluons in the intermediate state is

$f_{\mathrm{DC}}=2\left(v_{13}+v_{24}\right)\left(v_{12}+v_{34}\right)$.

Summing these contributions we find

$$
\begin{aligned}
f^{(8)}= & A B+2\left(v_{23}+v_{14}\right)\left(v_{23}+v_{14}\right)-\left(v_{23}\right. \\
& \left.-v_{14}\right)\left(v_{23}-v_{14}\right) .
\end{aligned}
$$

Table 1 The coefficients $C_{I J}$ with redistribution of colour

\begin{tabular}{rrrr}
\hline 1 & 1 & -1 & -1 \\
1 & 1 & -1 & -1 \\
-1 & -1 & 2 & 4 \\
-1 & -1 & 4 & 2 \\
\hline
\end{tabular}

Restoring the BKP Green function, the result can be written as

$f_{k_{1}, k_{2}}^{(8)}=\sum_{I, J=1}^{4} C_{I J} v_{I}\left[G^{1243}\left(y^{\prime}-y^{\prime \prime}\right)+G^{1342}\left(y^{\prime}-y^{\prime \prime}\right)\right] v_{J}$,

where $I=$ (13), (24), (23), (14), $J=(12),(34)$, (23), (14) and coefficients $C_{I J}$ are given in Table 1 .

\subsection{Two interactions with direct transmission of colour}

Here we study the contribution in which one gluon is emitted from the upper interaction in Fig. 1-4 and the other from the lower interaction. As before the cut BKP blob will enter as a whole and we can study the case with $\tilde{G} \rightarrow 1$ and introduce the full $\tilde{G}$ into the final formulae. The diagrams illustrating the double inclusive cross section will be the same as in Fig. 9 in which one should suppress emission from the pomeron but assume instead that both interactions are opened, that is, with fixed rapidities and transverse momenta. The amplitude $F$ in this case can be written as

$F^{(9)}=P^{(12)}\left(Y-y_{1}\right) P^{(34}\left(Y-y_{1}\right) f_{k_{1}, k_{2}}^{(9)} P^{(12)}\left(y_{2}\right) P^{(34)}\left(y_{2}\right)$,

where in $f^{(9)}\left(k_{1}, k_{2}\right)$ the transferred momenta are fixed at $k_{1}$ for the upper interaction and $k_{2}$ for the lower one. The final form of $f^{(9)}$ is determined after the study of the double diffractive (DD, Fig. 9-1), S and DC configurations. Again we have to retain only diagrams with two gluons in the intermediate state. 
Table 2 The coefficients $\tilde{C}_{I J}$ with direct colour transmission

\begin{tabular}{rrrr}
\hline 4 & 6 & -3 & -3 \\
6 & 4 & -3 & -3 \\
-3 & -3 & 4 & 6 \\
-3 & -3 & 6 & 4 \\
\hline
\end{tabular}

We find

$$
\begin{aligned}
f_{\mathrm{DD}}^{(9)}= & 2\left(v_{13}+v_{24}-v_{23}-v_{14}\right)\left(v_{13}+v_{24}-v_{23}-v_{14}\right), \\
f_{S}^{(9)}= & -\frac{1}{2}\left\{\left(v_{13}-v_{14}\right)\left(v_{13}-v_{14}\right)+\left(v_{24}-v_{14}\right)\left(v_{24}-v_{14}\right)\right. \\
& \left.+\left(v_{23}-v_{24}\right)\left(v_{23}-v_{24}\right)+\left(v_{23}-v_{13}\right)\left(v_{23}-v_{13}\right)\right\}, \\
f_{\mathrm{DC}}^{(9)}= & \left(v_{23}+v_{14}\right)\left(v_{23}+v_{14}\right)+\left(v_{24}+v_{13}\right)\left(v_{24}+v_{13}\right) .
\end{aligned}
$$

Summing these contributions and restoring the BKP Green functions we find

$f_{k_{1}, k_{2}}^{(9)}=\frac{1}{2} \sum_{I, J=1}^{4} \tilde{C}_{I J} v_{I} \tilde{G}\left(y^{\prime}-y^{\prime \prime}\right) v_{J}$,

where $I, J=(13),(24),(23),(14)$ and coefficients $\tilde{C}_{I J}$ are given in Table 2.

4.3 One gluon from the interaction, the other from the BKP state. Redistribution of colour

The amplitude $F$ in this case can be written as

$$
F^{(10)}=P^{(12)}\left(Y-y_{1}\right) P^{(34}\left(Y-y_{1}\right) f_{k_{1}, k_{2}}^{(10)} P^{(13)}\left(y_{2}\right) P^{(24)}\left(y_{2}\right) \text {. }
$$

It can be illustrated by the same diagrams as in Fig. 10 with the emission of the pomeron suppressed but instead one of the two interactions opened. We have two possibilities: either the gluon of higher rapidity is emitted by the upper interaction and the one of lower rapidity from the BKP state and vice versa.

We start from the first case. All we have to do is to take the known inclusive cross sections from the upper interaction and combine them with also known inclusive cross sections from the BKP state. However, we have to do it separately for each configuration of the cutting plane and also take into account the symmetry factors and the minus factor for the single cut for the contribution for a given configuration as a whole.

In the DT configuration from the interaction and from the BKP state we have, respectively, $V_{\mathrm{DT}}=2\left(v_{23}+v_{14}\right) B$ and $\mathrm{BKP}_{\mathrm{DT}}=v_{13}+v_{24}$. This gives a contribution to $f$ $f_{\mathrm{DT}}=2\left(v_{13}+v_{24}\right) G\left(v_{13}+v_{24}\right) G B$ where $G$ is either $G^{1243}$ or $G^{1342}$ with weight $1 / 2$. Suppressing the common right factor $G B$ and the remaining Green function we rewrite it as $f_{\mathrm{DT}}=2\left(v_{13}+v_{24}\right)\left(v_{13}+v_{24}\right)$. In the DP configuration from the interaction and from the BKP state we
Table 3 The coefficients $D_{I J}^{h}$ with redistribution of colour

\begin{tabular}{rrrr}
\hline 1 & 1 & 1 & 1 \\
1 & 1 & 1 & 1 \\
-3 & -2 & -3 & 0 \\
-3 & 0 & -3 & -2 \\
\hline
\end{tabular}

Table 4 The coefficients $D_{I J}^{l}$ with redistribution of colour

\begin{tabular}{rrrr}
\hline 1 & 1 & 0 & -2 \\
1 & 1 & -3 & -3 \\
1 & 1 & -2 & 0 \\
1 & 1 & -3 & -3 \\
\hline
\end{tabular}

have, respectively, $V_{\mathrm{DP}}=A B$ and $\mathrm{BKP}_{\mathrm{DP}}=v_{12}+v_{34}$. This gives a contribution $f_{\mathrm{DP}}=2 A\left(v_{12}+v_{34}\right)$. The single cut contribution from the interaction (without the minus sign) is $V_{S}=-\left(v_{13}-v_{14}\right) B$ and from the BKP state $\mathrm{BKP}_{S}=v_{12}+v_{13}$. This gives a contribution for a given choice of participants $S=\left(v_{13}-v_{14}\right)\left(v_{12}+v_{13}\right)$. The total contribution from the $\mathrm{S}$ configuration will be given by

$$
\begin{aligned}
f_{S}= & \left(v_{13}-v_{14}\right)\left(v_{12}+v_{13}\right)+\left(v_{13}-v_{23}\right)\left(v_{13}+v_{34}\right) \\
& +\left(v_{24}-v_{23}\right)\left(v_{12}+v_{24}\right)+\left(v_{24}-v_{14}\right)\left(v_{24}+v_{34}\right) .
\end{aligned}
$$

Finally the DC contribution from the interaction and the BKP state is, respectively, $V_{\mathrm{DC}}=-\left(v_{13}+v_{24}\right) B$ and $\mathrm{BKP}_{\mathrm{DC}}=$ $v_{\mathrm{BKP}}^{1243}$, so the total contributions is $f_{\mathrm{DC}}=-\left(v_{13}+v_{24}\right) v_{\mathrm{BKP}}^{1243}$.

In the sum the total double inclusive $f^{10, h}$ can be written as

$f_{k_{1}, k_{2}}^{10, h}=\frac{1}{2} \sum_{a} \sum_{I, J=1}^{4} D_{I J}^{h} v_{\mathrm{IG}}^{a} v_{\mathrm{JG}}^{a} B$,

where $a=1243,1342, I=$ (23), (14), (13), (24), $J=$ (12), (24), (34), (13) and the coefficients $D^{h}$ are given in Table 3.

The case of the gluon of the lower rapidity emitted from the interaction is considered quite similarly. The corresponding double inclusive function $f^{10, l}$ can be written as

$f_{k_{1}, k_{2}}^{10, l}=\frac{1}{2} \sum_{a} \sum_{I, J=1}^{4} D_{I J}^{l} \mathrm{AG}^{a} v_{\mathrm{IG}}^{a} v_{J}$

where $a=1243,1342, I=(12),(24),(34),(13), J=$ (23), (14), (12), (34) and the coefficients $D_{h}$ are given in Table 4.

In the end the total inclusive function $F^{(10)}$ will be given by (52) with $f^{(10)}=f^{10, h}+f^{10, l}$.

4.4 One gluon from the interaction, the other from the BKP state. Direct transmission of colour

The amplitude $F$ in this case can be written in the form $F^{(11)}=P^{(12)}\left(Y-y_{1}\right) P^{(34}\left(y-y_{1}\right) f_{k_{1}, k_{2}}^{(11)} P^{(12)}\left(y_{2}\right) P^{(34)}\left(y_{2}\right)$ 
Table 5 The coefficients $\tilde{D}_{I J}^{h}$ with direct transmission of colour

\begin{tabular}{rrrr}
\hline 2 & 2 & 2 & 2 \\
1 & 2 & 1 & 0 \\
1 & -2 & 0 & -1 \\
0 & -2 & 1 & -1 \\
\hline
\end{tabular}

The relevant BKP Green function is one of $G^{1234}, G^{1432}$, $G^{1243}$ and $G^{1342}$ with weight 1/4. Again we have two possibilities: either the gluon of higher rapidity is emitted by the upper interaction and the one of lower rapidity from the BKP state and vice versa. We start from the first case.

We find for the DD contribution from the interaction $V_{\mathrm{DD}}=A A$ and from the $\mathrm{BKP}$ state $\mathrm{BKP}=v_{14}+v_{23}$. Using our previous notations and suppressing the right $G A$ we have the contribution $f_{\mathrm{DD}}=2 A\left(v_{14}+v_{23}\right)$. For the $\mathrm{S}$ contribution we find from the interaction $V_{S}=-\left(v_{13}-v_{14}\right)$ (without the minus sign). From the BKP state we find BKP $=v_{12}+v_{14}$, which gives the contribution to $f$

$f_{S}=\left(v_{13}-v_{14}\right)\left(v_{12}+v_{14}\right)+\left(v_{24}-v_{14}\right)\left(v_{34}+v_{14}\right)$.

Finally for the DC contribution we find from the interaction $V_{\mathrm{DC}}=v_{23}+v_{14}$ and from the BKP state $\mathrm{BKP}=v_{12}+v_{34}$, which gives the contribution to $f f_{\mathrm{DC}}=2\left(v_{23}+v_{14}\right)\left(v_{12}+\right.$ $\left.v_{34}\right)$.

Summing all the contributions we find

$f_{k_{1}, k_{2}}^{11, h}=\frac{1}{4} \sum_{a} \sum_{I, J=1}^{4} \tilde{D}_{I J}^{h} v_{\mathrm{IG}}^{a} v_{\mathrm{JG}}^{a} A$

where $a=1234,1432,1243,1342, I=(23),(14),(13)$, (24), $J=(12),(23),(34),(14)$ and the coefficients $\tilde{D}_{h}$ are given in Table 5 .

In the case when the gluon of the lower rapidity is emitted from the interaction we similarly find

$f_{\mathrm{DD}}=2\left(v_{14}+v_{23}\right) A$,

$f_{S}=\left(v_{12}+v_{14}\right)\left(v_{13}-v_{14}\right)+\left(v_{34}+v_{14}\right)\left(v_{24}-v_{14}\right)$,

$f_{\mathrm{DC}}=2\left(v_{12}+v_{34}\right)\left(v_{23}+v_{14}\right)$.

Summed this gives

$f_{k_{1}, k_{2}}^{11, l}=\frac{1}{4} \sum_{a} \sum_{I, J=1}^{4} \tilde{D}_{I J}^{l} \mathrm{AG} v_{\mathrm{IG}} v_{J}$,

where $a=1234,1432,1243,1342, I=(12),(23)$, (34), (24), $J=(23),(14),(13),(24)$, and the coefficients $\tilde{D}_{l}$ are given in Table 6.

The final inclusive function $F^{(11)}$ will be given by (55) with $f^{(11)}=f^{11, h}+f^{11, l}$.
Table 6 The coefficients $\tilde{D}_{I J}^{l}$ with direct transmission of colour

\begin{tabular}{rrrr}
\hline 2 & 1 & 1 & 0 \\
2 & 2 & -2 & -2 \\
2 & 1 & 0 & 1 \\
2 & 0 & -1 & -1 \\
\hline
\end{tabular}

4.5 Both interactions from the BKP state

When both gluons are emitted from the BKP state, similarly to the BFKL pomeron, we have to 'open' it twice, that is, change

$G \rightarrow G f_{y_{1}, k_{1}} G f_{y_{2}, k_{2}} G$.

It is important that the cutting plane cannot change its position inside the BKP state, so that function $f$ has to be the same in both 'openings', but different for different cuts. The double inclusive cross section is illustrated by typical diagrams shown in Fig. 11.

Let us first consider the case of colour redistribution. The relevant BKP Green function is one of $G^{1243}$ and $G^{1342}$.

The amplitude $F$ in this case can be written as

$$
\begin{aligned}
F^{(12)}= & P^{(12)}\left(Y-y_{1}\right) P^{(34}\left(y-y_{1}\right) A f_{k_{1}, k_{2}}^{(12)} \\
& \times B P^{(13)}\left(y_{2}\right) P^{(24)}\left(y_{2}\right)
\end{aligned}
$$

In the DT configuration we find the 'opened' Green function

$f_{\mathrm{DT}}=2 G\left(v_{12}+v_{34}\right) G\left(v_{12}+v_{34}\right) G$.

In the DP configuration

$f_{\mathrm{DP}}=2 G\left(v_{13}+v_{24}\right) G\left(v_{13}+v_{24)} G\right.$.

In the $\mathrm{S}$ configuration

$$
\begin{aligned}
f_{S}= & -G\left(v_{12}+v_{13}\right) G\left(v_{12}+v_{13}\right) G-G\left(v_{13}+v_{34}\right) \\
& \times G\left(v_{13}+v_{34}\right) G-G\left(v_{12}+v_{24}\right) G\left(v_{12}+v_{24}\right) G \\
& -G\left(v_{34}+v_{24}\right) G\left(v_{34}+v_{24}\right) G .
\end{aligned}
$$

In the DC configuration

$f_{\mathrm{DC}}=2 G v_{\mathrm{BKP}}^{1243} v_{\mathrm{BKP}}^{1243} G$.

The final $f$ can be presented as

$f_{k_{1}, k_{2}}^{(12)}=\frac{1}{2} \sum_{a} \sum_{I, J=1}^{4} E_{I J} G^{a} v_{\mathrm{IG}}^{a} v_{\mathrm{JG}}^{a}$,

where $a=1243,1342, I, J=(12),(24),(34),(13)$ and the coefficients $E$ are given in Table 7 .

Now the case of direct colour transmission. The BKP Green function $G$ is one of four $G^{1234}, G^{1432}, G^{1243}$ and $G^{1342}$ with weight $1 / 4$. 
Fig. 11 Double gluon production from the BKP state in the diffractive (1), single (2) and double (2) cut configurations
Table 7 The coefficients $E_{I J}$ with redistribution of colour

Table 8 The coefficients $\tilde{E}_{I J}$ with direct transmission of colour

$$
\begin{aligned}
F^{(13)}= & \int \mathrm{d} \tau P^{(12)}\left(Y-y_{1}\right) P^{(34}\left(y-y_{1}\right) A f_{k_{1}, k_{2}}^{(13)} \\
& \times A P^{(12)}\left(y_{2}\right) P^{(34)}\left(y_{2}\right) .
\end{aligned}
$$

In the DD configuration we find the 'opened' Green function

$f_{\mathrm{DD}}=2 G\left(v_{14}+v_{23}\right) G\left(v_{14}+v_{23}\right) G$.

In the $\mathrm{S}$ configuration

$$
\begin{aligned}
f_{S}= & -G\left(v_{12}+v_{14}\right) G\left(v_{12}+v_{14}\right) G \\
& -G\left(v_{14}+v_{34}\right) G\left(v_{14}+v_{34}\right) G .
\end{aligned}
$$

In the DC configuration

$\left.f_{\mathrm{DC}}=2 G\left(v_{12}+v_{34}\right) v_{12}+v_{34}\right) G$.

The final $f$ can be presented as

$f_{k_{1}, k_{2}}^{(13)}=\frac{1}{4} \sum_{a} \sum_{I, J=1}^{4} \tilde{E}_{I J} G^{a} v_{\mathrm{IG}}^{a} v_{\mathrm{JG}}^{a}$

where $a=1234,1432,1243,1342, I, J=(12)$, (23), (34), (14) and the coefficients $\tilde{E}$ are given in Table 8 .
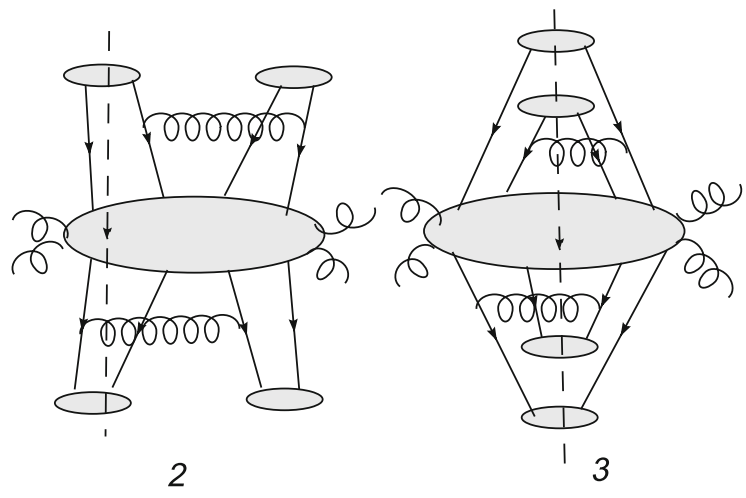

\section{Conclusions}

We have calculated the non-trivial part of the double inclusive cross section to produce two gluon jets in collision of two projectiles on two targets. It consists of 13 terms

$F=\sum_{i=1}^{13} F^{(i)}$

where particular terms are given by (20), (28), (31), (37), (40), (44), (45), (49), (50), (52), (55), (59) and (61).

This expression is not to be considered too complicated in view of variety of different possibilities for gluon emission. By its structure our theory is very similar to the old ReggeGribov model with a local pomeron and three-pomeron interaction generalised to include four-pomeron interactions (diagrams in Fig. 1-1,2) and a new pomeron-like object, the BKP state made of four reggeised gluons, with transitions to it from normal pomerons. Obviously the two gluons can be emitted from old and new pomerons and also from the two transition vertices. This gives rise to a multitude of contributions, which lead to (63).

The found inclusive cross section depends on three rapidities, the overall one $Y$ and two rapidities $y_{1}>>y_{2}$ of the observed gluons. All contributions have the same order $\alpha_{s}^{2} f\left(\alpha_{s} N_{c} Y, \alpha_{s} N_{c} y\right)$ where $y=y_{1}-y_{2}$ and $\alpha_{s} N_{c} Y \sim$ $\alpha_{s} N_{c} y \sim 1$. If one assumes the standard behaviour of the BFKL pomeron at large $Y$ in accordance with the BFKL equation, that is, roughly as $\sim \exp \left(Y \Delta_{\mathrm{BFKL}}\right)$ where $\Delta_{\mathrm{BFKL}}=4 \ln 2 \alpha_{s} N_{c} / \pi$ then all our contributions obviously grow as the pomeron squared, namely, as $\sim \exp \left(2 Y \Delta_{\text {BFKL }}\right)$. In this limit the BKP state appears at finite rapidities, since it grows much slower, as $\sim \exp \left(Y \Delta_{\mathrm{BKP}}\right)$ where $\Delta_{\mathrm{BKP}}=$ $0.243 \Delta_{\mathrm{BFKL}}$ [6]. As to the dependence on $y=y_{1}-y_{2}$ then if one or both gluons are emitted from pomerons then the contribution will grow as $\sim \exp y \Delta_{\text {BFKL. Otherwise the }}$ growth will be much weaker, as $\sim \exp y \Delta_{\text {BKP. Obviously }}$ the first type of contributions will dominate.

However, these estimates may be changed if one introduces damping of the pomeron growth at high energies 
either by using the experimental behaviour of the hadronic cross sections or the unitarisation procedure following the Balitski-Kovchegov equation $[7,8]$. Then the dominant contributions will come from the BKP state with a much weaker growth and maybe be available for experimental observation unless the latter contributions will also be damped by absorptive corrections similarly to the pomeron case.

Our contribution is of interest in view of recent experimental results on long-range rapidity and azimuthal correlations measured in experiments on colliders in nucleus-nucleus, proton-nucleus and proton-proton collisions. The immediate application of our results may be to deuteron-deuteron collisions.

An old question is whether the strong azimuthal decorrelation found in the strict application of the BFKL equation to dijet production (Mueller-Navelet jets [9]) is softened when the equation is generalised to include new contributions or higher order corrections. In particular in [10] it was found that next-to-leading order corrections to the BFKL equation, which are known to be quite large, drastically diminish decorrelation, so that the azimuthal asymmetry in dijet production remains well preserved up to rapidity distance $y=10$. In our paper we have actually studied a different sort of corrections, of the leading order in $\alpha_{s} N_{c}$ but subdominant in $1 / N_{c}^{2}$, which involve quite different diagrams. Staying at leading order in $\alpha_{s} N_{c}$ we find that all parts of the double inclusive cross section which involve emission from the pomeron will damp azimuthal asymmetry exactly as for the simple BFKL chain, that is, roughly as $\exp \left(-y \Delta_{\mathrm{BFKL}}\right)$. The new element is, however, inclusion of the intermediate BKP state whose ground state has a much smaller intercept $\Delta_{\mathrm{BKP}}$. Since this ground state is non-degenerate it is azimuthally symmetric. If, as with the BFKL pomeron, the lowest intercept of the azimuthally anisotropic states lies around zero then azimuthal decorrelation of jet pairs emitted from the BKP state will be much weaker than from the BFKL pomeron. In fact with $\Delta_{\mathrm{BFKL}}=0.3$ the decorrelation factor $\exp \left(-y \Delta_{\mathrm{BKP}}\right)$ is around 0.5 even at rapidity distance as high as $y=10$. This effect can be traced experimentally even if the relative contribution from the BKP state is small. Naturally this conclusion has to be checked by the study of the spectrum of the BKP states with non-zero angular momentum, which is unknown at present. This problem is postponed for future investigation. As mentioned in the Introduction the origin of azimuthal asymmetry in nucleus-nucleus collisions due to diagrams like Fig. 1-3,4 was considered in [5]. However, the possibility of the intermediate BKP state between the interactions and the following (possibly weak) azimuthal decorrelation was not taken into account there.

Acknowledgments This work has been supported by the RFFI Grant 12-02-00356-a and the SPbSU Grants 11.059.2010, 11.38.31.2011 and 11.38.660.2013. The author is indebted to J. Kotanski for discussions about the properties of the BKP states.

Open Access This article is distributed under the terms of the Creative Commons Attribution License which permits any use, distribution, and reproduction in any medium, provided the original author(s) and the source are credited.

Funded by $\mathrm{SCOAP}^{3}$ / License Version CC BY 4.0.

\section{References}

1. M.A. Braun, Eur. Phys. J. C 73, 2418 (2013). arXiv:1301.4846 [hep-ph]

2. M.A. Braun, Eur. Phys. J. C 73, 2511 (2013). arXiv:1305.1712 [hep-ph]

3. J. Bartels, Nucl. Phys. B 175, 365 (1980)

4. J. Kwiecinski, M. Praszalowicz, Phys. Lett. B 94, 413 (1980)

5. A. Dumitru, J. Jalilian-Marian, Phys. Rev. D 81, 094015 (2010)

6. G.P. Korchemsky, J. Kotansky, A.N. Manashov, Phys. Rev. Lett. 88, 122002 (2002)

7. I. Balitski, Nucl. Phys. B 463, 99 (1996)

8. Yu. Kovchegov, Phys. Rev. D 60, 034008 (1999)

9. A.H. Mueller, H. Navelet, Nucl. Phys. B 282, 727 (1987)

10. D. Colferai, F. Schwennsen, L. Szymanovski, S. Wallon, JHEP 1012, 026 (2010) 